\title{
Sepse puerperal: Uma revisão integrativa
}

\author{
Puerperal sepsis: An integrative review \\ Sepsis puerperal: Una revisión integradora
}

Recebido: 19/06/2021 | Revisado: 25/06/2021 | Aceito: 29/06/2021 | Publicado: 13/07/2021

\author{
Antonio Paulo Nunes da Silva \\ ORCID: https://orcid.org/0000-0003-1925-015X \\ Universidade de Pernambuco, Brasil \\ E-mail: paulonunes14081996@gmail.com \\ Pollyana Rodrigues Diniz \\ ORCID: https://orcid.org/0000-0003-2419-3401 \\ Universidade de Pernambuco, Brasil \\ E-mail: pollyrodrigues386@gmail.com \\ Polyana Felipe Ferreira da Costa \\ ORCID: https://orcid.org/0000-0002-6054-8401 \\ Universidade de Pernambuco, Brasil \\ E-mail: polyana.costa@upe.br \\ Pauliana Valéria Machado Galvão \\ ORCID: https://orcid.org/0000-0002-4418-218X \\ Universidade de Pernambuco, Brasil \\ E-mail: pauliana.galvao@upe.br \\ Valda Lúcia Moreira Luna \\ ORCID: https://orcid.org/0000-0002-1810-7565 \\ Universidade de Pernambuco, Brasil \\ E-mail: valda.moreira@upe.br \\ George Alessandro Maranhão Conrado \\ ORCID: https://orcid.org/0000-0001-6649-577X \\ Universidade de Pernambuco, Brasil \\ E-mail: george.maranhao@upe.br
}

\begin{abstract}
Resumo
Em 2016, o conceito de sepse foi redefinido, entretanto ainda carece de melhor caracterização. No Brasil, esta complicação apresenta mortalidade de $28 \%$ e gastos de aproximadamente 9,1 mil dólares por paciente. Diante das dificuldades de definição e diagnóstico, este trabalho se propõe a sintetizar evidências científicas disponíveis. Trata-se de uma revisão integrativa realizada entre janeiro e junho de 2021, para responder "Quais são as evidências recentes acerca da sepse puerperal?". A busca considerou os últimos 5 anos e resultou em 53 artigos, dos quais 14 foram selecionados devido aos critérios de exclusão. Destes, 28,6\% são diretrizes, 64,3\% estudos observacionais e 7,1\%, experimentais. Em relação aos países de publicação, mais de 50\% foram realizados nos EUA (35,75\%) e Inglaterra $(14,3 \%)$. Quanto ao idioma das publicações, 78,5\% estavam redigidos na língua inglesa; 14,3\%, na língua espanhola, e um 7,2\% na língua portuguesa. A sepse puerperal mantém-se como uma das principais causas de morbidade e mortalidade em todo o mundo. A fisiopatologia não é completamente compreendida e faltam evidências científicas, sobretudo no tocante ao diagnóstico e tratamento precoces. Em relação ao diagnóstico, as alterações fisiológicas da gravidez acabam por mascarar o quadro clínico e tornar a investigação desafiadora. Assim, anamnese e exame físico continuam imprescindíveis, auxiliados por exames complementares. O tratamento objetiva o reconhecimento precoce e a intervenção agressiva, por meio da antibioticoterapia, reposição de fluidos e suporte cardiovascular.
\end{abstract}

Palavras-chave: Sepse; Período pós-parto; Infecção puerperal.

\begin{abstract}
In 2016, the concept of sepsis was redefined, however it still needs to be better characterized. In Brazil, this complication has a mortality of $28 \%$ and costs of approximately 9,100 dollars per patient. Given the difficulties of definition and diagnosis, this work aims to synthesize available scientific evidence. This is an integrative review carried out between January and June 2021, to answer "What is the recent evidence about puerperal sepsis?". The search considered the last 5 years and resulted in 53 articles, of which 14 were selected due to the exclusion criteria. Of these, $28.6 \%$ are guidelines, $64.3 \%$ are observational studies and $7.1 \%$ are experimental. Regarding the countries of publication, more than $50 \%$ were carried out in the USA $(35.75 \%)$ and England $(14.3 \%)$. As for the language of the publications, 78.5\% were written in English; 14.3\% in Spanish, and 7.2\% in Portuguese. Puerperal sepsis remains one of the main causes of morbidity and mortality worldwide. The pathophysiology is not fully understood, and scientific evidence is lacking, especially regarding early diagnosis and treatment. Regarding the diagnosis, the physiological changes of pregnancy end up masking the clinical picture and making the investigation challenging. Thus, anamnesis
\end{abstract}


and physical examination are still essential, aided by complementary exams. Treatment aims at early recognition and aggressive intervention through antibiotic therapy, fluid replacement and cardiovascular support.

Keywords: Sepsis; Postpartum period; Puerperal infection.

\section{Resumen}

En 2016, se redefinió el concepto de sepsis, sin embargo, aún debe caracterizarse mejor. En Brasil, esta complicación tiene una mortalidad del $28 \%$ y un costo de aproximadamente 9.100 dólares por paciente. Dadas las dificultades de definición y diagnóstico, este trabajo tiene como objetivo sintetizar la evidencia científica disponible. Se trata de una revisión integradora realizada entre enero y junio de 2021, para responder "¿Cuál es la evidencia reciente sobre la sepsis puerperal?". La búsqueda consideró los últimos 5 años y resultó en 53 artículos, de los cuales 14 fueron seleccionados por criterios de exclusión. De estos, el 28,6\% son guías, el $64,3 \%$ estudios observacionales y el 7,1\% experimentales. En cuanto a los países de publicación, más del 50\% se realizaron en EE.UU. (35,75\%) e Inglaterra $(14,3 \%)$. En cuanto al idioma de las publicaciones, el 78,5\% fueron redactadas en inglés; $14,3 \%$ en español y 7,2\% en portugués. La sepsis puerperal sigue siendo una de las principales causas de morbilidad y mortalidad en todo el mundo. La fisiopatología no se comprende completamente y se carece de evidencia científica, especialmente en lo que respecta al diagnóstico y tratamiento precoces. En cuanto al diagnóstico, los cambios fisiológicos del embarazo terminan enmascarando el cuadro clínico y dificultando la investigación. Por tanto, la anamnesis y la exploración física siguen siendo imprescindibles, con la ayuda de exploraciones complementarias. El tratamiento tiene como objetivo el reconocimiento temprano y la intervención agresiva a través de la terapia con antibióticos, la reposición de líquidos y el apoyo cardiovascular.

Palabras clave: Sepsis; Período posparto; Infección puerperal.

\section{Introdução}

Em 2016, o Terceiro Consenso Internacional de Definições de Sepse e de Choque Séptico redefiniu sepse como um estado de disfunção orgânica generalizada com risco de vida decorrente de uma etiologia infecciosa (Singer et al., 2016; Belarmino et al., 2020; Foeller et al., 2020). Sobre a sepse materna, a Organização Mundial de Saúde (OMS) afirma tratar-se de disfunção orgânica resultante de infecção durante a gravidez, parto ou período pós-parto (Belarmino et al., 2020). Dentro dessa classificação, a sepse puerperal apesar de se tratar de condição crítica e com risco de vida, carece de melhor caracterização a fim de reconhecimento precoce (Foeller et al., 2020).

De modo geral, a infecção relacionada à gravidez é a terceira causa de morte materna no mundo e nos Estados Unidos da América (EUA), com mortalidade quase constante desde 1990 (Foeller et al., 2020; Phillips \& Walsh, 2020; Viderman et al., 2021). Em países em desenvolvimento, a incidência de sepse puerperal é estimada entre 0,1\% e 10\%, embora haja grande disparidade das estimativas devido à diferença nos critérios diagnósticos entre as fontes de estudo (Lopez \& Müller, 2016).

No Brasil, a incidência é estimada em $0,001 \%$ a $0,02 \%$ dos partos, com mortalidade de $28 \%$. É considerada, portanto, uma das cinco principais causas de morte materna no mundo, com maior frequência em países da Ásia, da África, da América Latina e Caribe. Além de altas taxas de morbimortalidade mundiais, tal problemática é responsável por gastos anuais de 20 bilhões de dólares nos EUA e de 9,1 mil dólares por paciente no Brasil (Belarmino et al., 2020).

A literatura traz como fatores de risco principais o parto operatório, além do parto prolongado, ruptura de membranas com várias horas de evolução, corioamnionite prévia, exames vaginais repetidos, infecções vaginais antes do parto e monitoramento fetal interno, entre outros (Céspedes-Fernández et al., 2020; Lopez \& Müller, 2016).

Durante a gestação, mudanças fisiológicas são perceptíveis em diversos sistemas. Nesse período, a progesterona estimula a vasodilatação materna; o parto e as dores pós-parto podem levar a taquicardia e a elevação do diafragma na gravidez e o aumento do consumo de oxigênio resulta em taquipneia leve. Além disso, a contagem de leucócitos é normalmente elevada durante a gravidez e no início do período pós-parto, tornando este um parâmetro de diagnóstico menos sensível. Em contribuição, a inflamação sistêmica no trabalho de parto e no período pós-parto inicial pode surgir secundária ao uso de prostaglandinas para indução do parto ou no tratamento da hemorragia pós-parto (Galvão et al., 2016; Matos et al., 2019; Phillips \& Walsh, 2020). Desse modo, o diagnóstico de sepse através do escore Sequential Organ Failure Assessment (SOFA) e o Quick Sequential Organ Failure Assessment (qSOFA) pode ser desafiador. 
Ainda, alguns sintomas iniciais, como dor abdominal, febre e mialgia podem ser inespecíficos e sutis (Morgan, 2020). Diante desse cenário de dificuldade na definição e na caracterização da sepse puerperal e escassez de evidências científicas, faz-se de suma importância a síntese sobre esse assunto à luz do conhecimento atual, objetivando a redução dessa condição potencialmente fatal por meio de detecção, diagnóstico e terapêutica precoces, bem como a implantação de políticas efetivas de promoção e prevenção em saúde voltadas às gestantes (Silva et al., 2020).

\section{Metodologia}

O presente estudo trata-se de uma revisão integrativa operacionalizada por meio das seguintes etapas: (1) formulação de questão de pesquisa; (2) estabelecimento de critérios de inclusão e exclusão; (3) coleta de dados; (4) avaliação dos estudos selecionados; (5) análise e interpretação dos resultados; e (6) apresentação da súmula do conhecimento (Mendes et al., 2008).

Nesse sentido, esta pesquisa objetivou responder ao seguinte questionamento norteador: "Quais são as evidências recentes disponíveis na literatura acerca da classificação e epidemiologia da sepse puerperal?”. Assim, no período de janeiro a junho do ano de 2021, publicações científicas indexadas foram pesquisadas, empregando as bases de dados Scientific Electronic Library Online (SciELO), Medical Literature Analysis and Retrievel System Online (Medline) e Literatura LatinoAmericana e do Caribe em Ciências da Saúde (LILACS). Para tanto, adotaram-se os Descritores em Ciências da Saúde (DECS) controlados: "sepsis" e "postpartum period", integrados pelo operador booleano AND.

Estabeleceram-se como critérios para a seleção da amostra: artigos completos que abordassem a temática pesquisada, publicados entre os anos de 2016-2021, sem restrição de idioma. Foram excluídos os estudos do tipo revisão sistemática e metanálise, uma vez que realizam, respectivamente, a descrição/método estatístico de análise de uma evidência já sumarizada/reunida sistematicamente (Baena, 2014), além de Editoriais e Cartas ao Editor. Também foram desconsiderados os estudos que não respondiam à questão norteadora e os que se repetiram nas bases de dados.

A busca inicial na literatura resultou em uma amostra de 53 publicações. À luz dos critérios de elegibilidade, 19 artigos foram pré-selecionados. Posteriormente, realizou-se a leitura na íntegra dos artigos, dos quais 14 foram incluídos por responderem satisfatoriamente à questão norteadora e, portanto, compuseram o conjunto final de análise desta revisão. O fluxograma com o detalhamento das etapas de seleção das publicações é apresentado na Figura 1.

Com vistas a assegurar os aspectos éticos inerentes a essa pesquisa, a autoria e as citações de cada publicação foram devidamente respeitadas por meio de sua referenciação. Cabe salientar ainda, que por se tratar de um estudo de revisão de literatura, e por conseguinte sem o envolvimento de seres humanos, é dispensada a apreciação pelo Comitê de Ética em Pesquisa segundo a Resolução 510/2016 do Conselho Nacional de Saúde. 
Figura 1. Fluxograma do processo de seleção dos estudos incluídos na revisão.
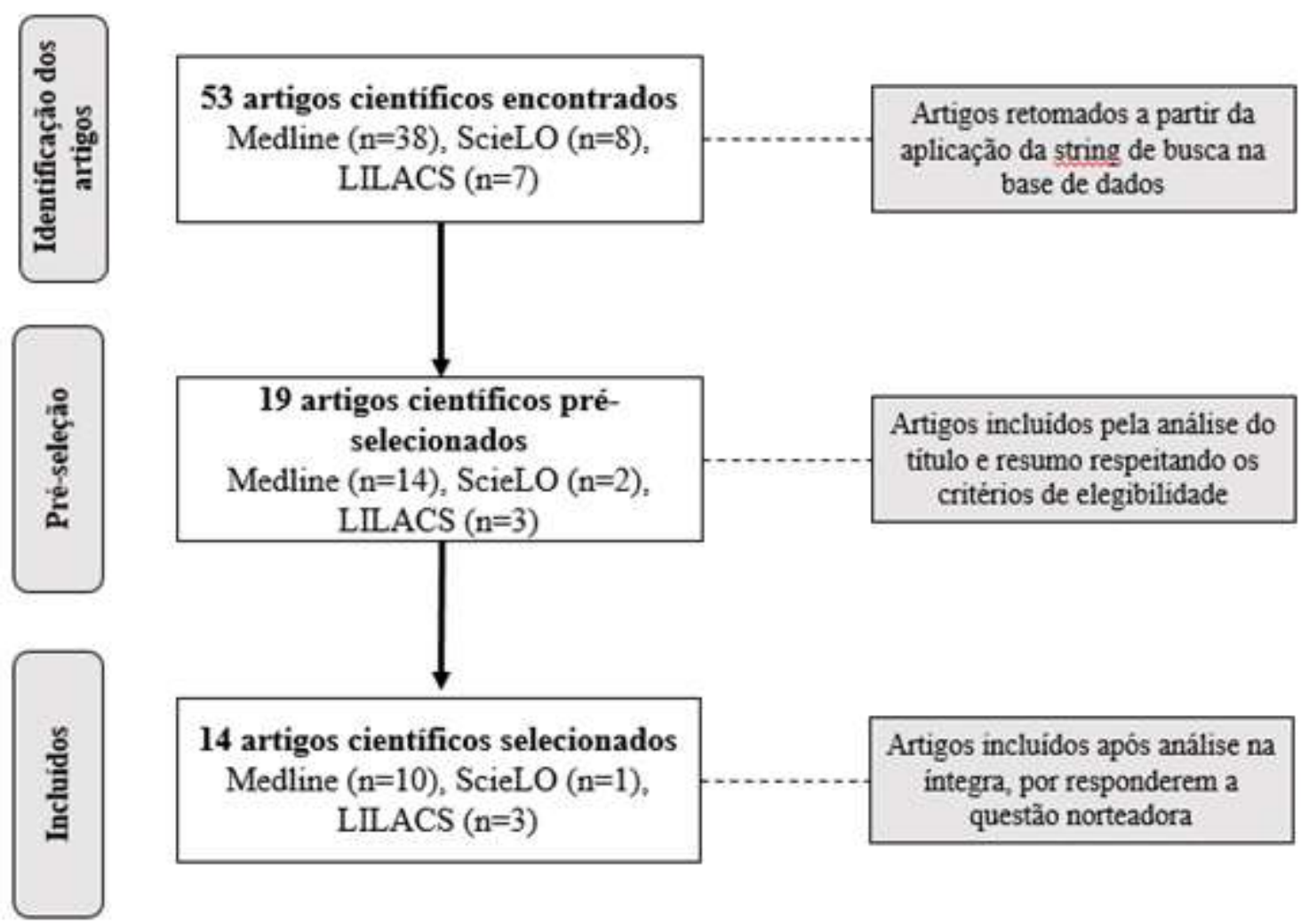

Fonte: Pesquisa própria (2021).

\section{Resultados}

Os artigos selecionados $(\mathrm{n}=14)$ foram identificados e apresentados, para análise crítica dos dados, conforme a organização descrita na Tabela 1 .

Tabela 1. Distribuição dos artigos incluídos na revisão integrativa segundo título, autores/ano, periódicos e objetivos.

\begin{tabular}{|c|c|c|c|}
\hline $\begin{array}{c}\text { Autores } \\
\text { (ano) }\end{array}$ & Objetivos & Resultados & Conclusões \\
\hline $\begin{array}{l}\text { Belarmino } \\
\text { et al. } \\
(2020)\end{array}$ & $\begin{array}{l}\text { Evidenciar as dificuldades na } \\
\text { identificação da sepse } \\
\text { materna pelos profissionais } \\
\text { de enfermagem. }\end{array}$ & $\begin{array}{l}\text { Foram evidenciadas dificuldades na identificação } \\
\text { da sepse materna, atraso no início da administração } \\
\text { de antibióticos e falta de comunicação entre as } \\
\text { categorias profissionais. }\end{array}$ & $\begin{array}{l}\text { O protocolo mostrou-se empregável, } \\
\text { apesar de fragilidades na } \\
\text { implementação. }\end{array}$ \\
\hline $\begin{array}{l}\text { Duan et al. } \\
\quad(2019)\end{array}$ & $\begin{array}{l}\text { Analisar o prognóstico de } \\
\text { mulheres grávidas com sepse } \\
\text { bacteriana que foram } \\
\text { admitidas em um centro de } \\
\text { cuidados terciários no oeste } \\
\text { da China. }\end{array}$ & $\begin{array}{l}\text { Entre as mulheres grávidas com sepse bacteriana, a } \\
\text { infecção do trato genital foi a fonte mais comum de } \\
\text { infecção. A bactéria mais comum no grupo de } \\
\text { bacterias Gram-positivas foi Listeria } \\
\text { monocytogenes e no grupo de bactérias Gram- } \\
\text { negativas foi Escherichia coli. As taxas de partos } \\
\text { prematuros foram substancialmente maior no grupo } \\
\text { de bactérias Gram-negativas, e a taxa de aborto foi } \\
\text { maior no grupo de bactérias Gram-positivas. }\end{array}$ & $\begin{array}{l}\text { A sepse bacteriana permanece uma } \\
\text { causa alarmante de morbidade e } \\
\text { mortalidade materna e fetal e } \\
\text { mortalidade infantil. O tratamento } \\
\text { chave envolve um grupo } \\
\text { multidisciplinar de médicos com } \\
\text { experiência em todos os aspectos do } \\
\text { atendimento a mulheres com sepse e } \\
\text { início precoce de antibióticos } \\
\text { apropriados de acordo com o tipo de } \\
\text { infecção bacteriana. }\end{array}$ \\
\hline
\end{tabular}


Cont.:Tabela 1. Distribuição dos artigos incluídos na revisão integrativa segundo título, autores/ano, periódicos e objetivos.

$\begin{array}{cccc}\begin{array}{c}\text { Autores } \\ (\text { ano })\end{array} & \text { Objetivos } & \text { Resultados } & \text { Conclusões }\end{array}$

Foeller et Identificar os fatores de risco, al. (2019) comparando características, diagnósticos e organismos infectantes de readmissão materna precoce a tardia com sepse.

Galvão et Descrever as definições, Foram evidenciadas a importância da definição da al. (2016) fisiopatologia, incidência, sepse, a fisiopatologia em cada sistema corporal, diagnóstico, manejo, suas etiologias obstétricas e não obstétricas, além tratamento, prevenção e do desenvolvimento de diretrizes para o tratamento evolução da sepse durante a da sepse materna e a criação de sistemas de gravidez e o período pós- pontuação específicos que constituem prioridades parto.

García et Apresentar um caso clínico al. (2016) sobre choque tóxico no puerpério, que evoluiu para choque séptico e disfunção múltipla de órgãos.

Koo Relatar um caso raro de

(2018) choque séptico complicado por fasceíte necrotizante após uma cesariana.

Knight et Investigar o potencial al. (2019) benefício na prevenção da sepse puerperal pelo uso de antibióticos profiláticos.

Lopez \& Apresentar um caso clínico Müller sobre sepse puerperal causada

(2016) por salpingite associada à peritonite pélvica.

Morgan Descrever as principais (2020) causas, reconhecimento, diferenciação e manejo microbiológico da sepse na gravidez, utilizando dois casos clínicos detalhados para ilustrar.
Os fatores de risco para readmissão com sepse incluíram nascimento prematuro, hemorragia, obesidade, seguro fornecido pelo governo e cesárea primária. Para readmissões de sepse precoce e tardia, os diagnósticos mais comuns foram infecção do trato urinário e pielonefrite, e o organismo infectante identificado com mais frequência foram bactérias gram-negativas. urgentes.

Paciente com diagnóstico de síndrome do choque tóxico no puerpério, que apresentou choque séptico e disfunção multiorgânica em seguida.

Mulher de 30 anos desenvolveu choque séptico puerperal e fasciíte necrosante por Escherichia coli e Staphylococcus caprae, uma bactéria comumente encontrada em animais.

Foi mostrado o benefício do uso de antibióticos de forma profilática após parto vaginal operatório.

Primigesta jovem diagnosticada com endometrite pós-parto, evoluindo com salpingite e peritonite.

Evidenciou a abordagem clínica das pacientes com sepse através de dois casos clínicos: uma grávida e uma puérpera que apresentaram sintomas e sinais clínicos de Síndrome do choque tóxico estafilocócico e Síndrome do choque tóxico estreptocócico, respectivamente. Com isso, foi debatido acerca da investigação e principais tratamentos utilizados.
Os fatores de risco maternos para readmissão precoce e tardia com sepse incluíram características demográficas, cesárea, hemorragia e parto prematuro. Os riscos de sepse após o parto persistem além do período pós-parto tradicional de 6 semanas.

É absolutamente necessário validar as definições de sepse e suas condições relacionadas à obstetrícia. Pesquisas futuras devem se concentrar no resultado fetal de gestantes em estado crítico e na criação de um sistema de pontuação que possa prever a deterioração clínica nessa população.

$\begin{array}{llll}\text { Destaca-se } & \text { a } & \text { importância de } \\ \text { diagnóstico } & \text { e } & \text { de intervenção } \\ \text { oportunas. } & & \end{array}$

Este é o primeiro relato de fasciíte necrosante causada por Staphylococcus caprae complicada por choque séptico puerperal.

As orientações da OMS e de outras organizações precisam ser revistas.

A peritonite é uma complicação séria e rara no período pós-parto, necessitando de diagnóstico e tratamento precoces.

Conclui-se os seguintes pontos práticos: sempre verificar os resultados com cuidado para garantir que os organismos anteriores sejam sensíveis aos antimicrobianos propostos, além disso a terapia dupla é geralmente necessária, pelo menos para começar, se uma infecção produtora de exotoxina for a causa da sepse.

Cont. $=$ continuação OMS $=$ Organização Mundial da Saúde . 
Cont.:Tabela 1. Distribuição dos artigos incluídos na revisão integrativa segundo título, autores/ano, periódicos e objetivos.

$\begin{array}{cccc}\begin{array}{c}\text { Autores } \\ (\text { ano })\end{array} & \text { Objetivos } & \text { Resultados } & \text { Conclusões }\end{array}$

Plante et Resumir o que se sabe

al. (2019) sobre a sepse e fornecer orientações para o manejo da sepse na gravidez e no período pós-parto.

Ramírez Caracterizar a microbiota Salinas et da infecção operatória em al. (2016) 69 puérperas submetidas ao parto cesáreo.

Shafik et Determinar a incidência da al. (2019) síndrome da resposta inflamatória sistêmica (SIRS) e sua associação com a sepse aguda no período pós-parto imediato em 638 puérperas.

Viderman Apresentar um caso et al. clínico de infecção (2021) relacionada à gravidez, que evoluiu para choque sépticoratário acompanhado de púrpura fulminante e falência de múltiplos órgãos.
As principais recomendações são: (1) sepse e choque séptico são emergências médicas e o tratamento e a reanimação deve ser iniciada imediatamente; (2) antibióticos empíricos de amplo espectro devem ser administrados idealmente dentro de 1 hora, em qualquer gestante com suspeita de sepse; (3) o controle inicial da fonte deve ser concluído o mais rápido possível; (4) a administração precoce de 1-2 L de soluções cristalóides e norepinefrina na sepse complicada.

Prevaleceram as infecções monomicrobianas por bactérias gram-positivas, como o Staphylococcus aureus. Agentes multidrogas resistentes também foram encontrados.

Foi descoberto que $72,10 \%$ dos sinais vitais de mulheres no pós-parto imediato atendiam aos critérios SIRS, enquanto apenas $1,25 \%$ tiveram sepse. Tanto a idade pré-gestacional menor que 37 semanas e apenas um dos quatro critérios SIRS de temperatura anormal foram cada um significativamente associado a chances aumentadas de sepse.

Puérpera jovem desenvolveu choque séptico fulminante complicado por púrpura fulminante e síndrome de insuficiência múltipla de órgãos. Apesar da mortalidade esperada ser acima de 90\%, a paciente sobreviveu.
A sepse continua a ser uma das principais causas de morbidade e mortalidade em todo o mundo. $\mathrm{O}$ tratamento durante a gravidez e puerpério deve seguir os mesmos princípios básicos da população não grávida, incluindo o reconhecimento precoce, reposição de fluidos, antibióticos de amplo espectro oportunos e controle de fonte.

A presença de fatores de risco modificáveis condiciona o surgimento de infecções, o que requer um pré-natal adequado.

A SIRS pós-parto imediata não é útil para a identificação de sepse pós-parto imediata. Além disso, a SIRS não parece ser uma ferramenta útil de triagem para infecção e sepse no período pós-parto imediato.

O choque séptico é uma condição ameaçadora à vida não completamente compreendida, podendo estar associada à púrpura fulminante, disfunção de múltiplos órgãos, coagulação intravascular disseminada e necrose maciça de tecido.

SIRS: síndrome da resposta inflamatória sistêmica. Fonte: Pesquisa Própria.

A disposição dos artigos selecionados quanto ao seu desenho metodológico demonstra que quatro $(28,6 \%)$ desses constituíram-se como diretrizes, nove $(64,3 \%)$ estudos observacionais e um $(7,1 \%)$ estudo experimental (ensaio clínico randomizado e controlado).

No que se refere aos países de origem dos estudos, constatou-se a seguinte distribuição: Estados Unidos 35,7\% (5), Inglaterra 14,3\% (2), Coréia do Sul 14,3\% (2), Brasil 7,2\% (1), Bulgária 7,2\% (1), Cuba 7,2\% (1), México 7,2\% (1) e Arábia Saudita 7,2\% (1). Porém, quanto ao idioma das publicações, onze (78,5\%) estavam redigidos na língua inglesa, dois (14,3\%), na língua espanhola e um $(7,2 \%)$ na língua portuguesa. Quanto ao ano de publicação das produções científicas, por buscar as evidências mais recentes, foram pesquisados artigos publicados nos últimos cinco anos (2016-2021). As publicações contemplaram periódicos diferentes, todos internacionais.

\section{Discussão}

A infecção puerperal é uma das principais complicações da paciente obstétrica e apesar dos importantes avanços da obstetrícia contemporânea, o conhecimento dos fatores de risco e o advento da de novos tratamentos, constitui uma das mais importantes causas da mortalidade materna. Nesse contexto, dos diversos tipos de infecção puerperal, destaca-se a sepse puerperal, uma condição infrequente, mas persistente e que apresenta dificuldades na sua definição (Ramírez Salinas et al., 
2016; Galvão et al., 2016).

Segundo Lopez e Muller (2016), a sepse puerperal era definida como a infecção ocorrida entre a ruptura das membranas e os primeiros 42 dias pós-parto, com pelo menos duas das seguintes condições: dor pélvica, febre - temperatura oral igual ou superior a $38,5^{\circ} \mathrm{C}$ - e corrimento vaginal purulento, turvo ou fétido ou involução uterina retardada. Entretanto, após uma definição revisada, segundo a Organização Mundial da Saúde, a sepse materna foi reclassificada como "disfunção orgânica resultante de infecção durante a gravidez, parto, pós-aborto ou período pós-parto”, incluindo assim, de maneira mais geral, a sepse puerperal. Dessa forma, a presente definição da sepse busca enfatizar mais os sinais de disfunção orgânica do que os sintomas de infecção (Lopez \& Müller, 2016; Plante et al., 2019; Belarmino et al., 2020; Foeller et al., 2020).

A fisiopatologia da sepse é complexa e não totalmente compreendida, além de apresentar vários desequilíbrios que afetam uma grande diversidade dos sistemas orgânicos. O estado hiperinflamatório que ocorre com sepse resulta em disfunção de órgão terminal que pode ser desencadeado em indivíduos imunossuprimidos ou estimulados por imunidade e inclui extravasamento de albumina e líquido, com hipovolemia intravascular resultante. Ocorre um desencadeamento generalizado de mediadores pró-inflamatórios e citocinas, bem como ativação do sistema complemento. Essa liberação de citocinas leva à diminuição da resistência vascular sistêmica e aumento do débito cardíaco, embora até $60 \%$ dos pacientes com sepse tenham uma fração de ejeção abaixo de 45\% (disfunção sistólica) (Galvão et al., 2016; Plante et al., 2019; Phillips \& Walsh, 2020).

Lesão celular generalizada pode causar distúrbios metabólicos, isquemia dos tecidos e déficits de coagulação que levam à coagulação intravascular disseminada e resulta em dano endotelial, vasodilatação e hipovolemia. Mulheres com sepse podem ser edematosas porque o aumento da permeabilidade capilar pode causar transferência de fluido para os espaços extravasculares; no entanto, elas são hipovolêmicas porque diminuíram o volume intravascular. A síndrome de disfunção de múltiplos órgãos resulta da perfusão tissular prejudicada e morte celular associada à sepse e grave inflamação. Os sistemas de órgãos comumente afetados são os sistemas respiratório, renal, hepático, gastrointestinal, cardiovascular e nervoso (Galvão et al., 2016; Plante et al., 2019; Phillips \& Walsh, 2020).

As alterações cardiovasculares normais da gravidez são semelhantes às que ocorrem na sepse materna e podem, portanto, mascarar sua apresentação inicial e agravar a perfusão dos órgãos. Conforme o choque séptico progride, sinais de hipoperfusão podem se desenvolver e reduzir o suprimento de oxigênio aos tecidos, levando ao metabolismo anaeróbico, acúmulo de lactato, diminuição da perfusão uterina, acidose fetal e falência de órgãos-alvo. Em relação ao sistema gastrointestinal, a hipoperfusão da mucosa gástrica que ocorre durante a sepse origina atrofia da mucosa, que leva à translocação bacteriana e exacerbação da doença (Galvão et al., 2016; Phillips \& Walsh, 2020).

Quanto ao sistema respiratório, há um aumento significativo na ventilação minuto, causando uma queda na pressão parcial de dióxido de carbono ( $\mathrm{PaCO} 2$ ), o que leva a uma alcalose respiratória compensada. Isso é benéfico em uma gravidez normal, mas é prejudicial no caso de evolução da sepse, porque predispõe ao rápido declínio da oxigenação e diminuição da capacidade de compensar uma acidose metabólica. As alterações renais, como o aumento do fluxo plasmático renal e a taxa de filtração glomerular durante a gravidez, resultando em níveis mais baixos de creatinina, o que em casos de sepse podem significar comprometimento renal leve (Galvão et al., 2016; Phillips \& Walsh, 2020).

As evidências existentes identificam vários fatores de risco para sepse puerperal. Cesárea, parto prolongado, ruptura de membranas com várias horas de evolução, corioamnionite prévia, exames vaginais repetidos, infecções vaginais antes do parto ou cesariana e monitoramento fetal interno são os principais fatores de risco dessa condição patológica. Desses, o mais importante é a cesariana, portanto, a administração de antibióticos profiláticos durante a cirurgia é de suma importância, visto que reduz substancialmente o risco de infecção. Em consonância com isso, o estudo de Knight et al. (2019) evidenciou que, sem profilaxia, cerca de 20-25\% das mulheres têm uma infecção após o parto cesáreo, e até $16 \%$ têm uma infecção após o parto vaginal operatório (Lopez \& Muller, 2016; Ramírez Salinas et al., 2016; Knight et al., 2019; Viderman et al., 2021). 
Também outros fatores de risco se destacam, como obesidade, parto vaginal operatório, idade inferior a 25 anos, multiparidade, anemia, diabetes, condição imunossuprimida, indução de parto e parto prematuro. A presença de alguns desses fatores de risco mostra sua influência no surgimento da infecção puerperal e a importância do controle pré-natal adequado nas gestantes (Lopez \& Muller, 2016; Ramírez Salinas et al., 2016; Koo, 2018).

Os agentes etiológicos da sepse puerperal podem incluir bactérias sexualmente transmissíveis, bactérias da microbiota endógena ao paciente ou associações de ambos. Entre as bactérias sexualmente transmissíveis, Chlamydia trachomatis, Neisseria gonorrhoeae e, menos frequentemente, Mycoplasma hominis e Mycoplasma genitalium estão incluídos. Entre as bactérias da microbiota endógena, Enterobacteriaceae, cocos gram-positivos, como Streptococcus spp. e Enterococcus faecalis, e bactérias anaeróbias estritas são encontradas. Entretanto, os organismos mais comuns associados à sepse puerperal são Eschericchia coli, Staphylococcus e Streptococcus. Infecções mistas também são possíveis; em 15\% das mortes por sepse materna em que os organismos podem ser identificados, as infecções polimicrobianas (Lopez \& Muller, 2016; Koo, 2018; Plante et al., 2019).

A sepse puerperal é diagnosticada clinicamente; todavia, investigações completas, incluindo estudos de imagem relevantes, hemoculturas e quaisquer outros testes baseados na suspeita clínica da origem da infecção, devem ser realizadas para a investigação diagnóstica. O início da sepse pode ser insidioso, principalmente no pós-parto, o que torna o diagnóstico mais difícil. Por outro lado, em alguns casos, a sepse obstétrica pode ser fulminante e rapidamente fatal (Galvão et al., 2016; Koo, 2018).

Uma história completa e um exame físico são a primeira etapa na avaliação de um paciente com possível sepse. Inicialmente, os sinais clínicos variam de acordo com o local da infecção. Sinais como febre, hipotermia, taquipneia, taquicardia, hipotensão, oligúria, comprometimento da consciência e sensibilidade abdominal ou pélvica são sugestivos de sepse na população obstétrica. Um dos primeiros sinais clínicos de sepse é a taquipneia, que surge de pirexia, acidose láctica ou efeitos mediados por citocinas no centro respiratório. O diagnóstico pode ser desafiador porque os sintomas iniciais são frequentemente tênue, e embora as mulheres no período pós-parto corram maior risco de infecção, é relativamente incomum. A dor abdominal pode ser atribuída a dores pós-parto ou pós-operatória. Anestesia e analgesia podem mascarar os primeiros sinais de infecção e sepse, como dor e febre (Galvão et al., 2016; Phillips \& Walsh, 2020).

Em relação às análises laboratoriais, utilizar a contagem de leucócitos como indicativo de sepse é imprudente, pois as gestantes tendem a apresentar níveis mais elevados dessas células. A obtenção de hemoculturas é fundamental para a investigação diagnóstica e, idealmente, deve ser realizada antes da administração do antibiótico. Se houver suspeita clínica do foco de infecção, culturas de locais adicionais devem ser obtidas. Sendo assim, devem ser colhidos dois conjuntos de hemoculturas (com frascos aeróbicos e anaeróbicos), juntamente com quaisquer outras culturas apropriadas, como urina, ferida, tecido, escarro e líquido amniótico. Da mesma forma, quaisquer estudos de imagem relevantes devem ser prontamente realizados a fim de identificar a fonte da infecção (Galvão et al., 2016; Koo, 2018; Phillips \& Walsh, 2020).

Para auxiliar na avaliação da suspeita de sepse, vários sistemas de pontuação foram concebidos para orientar os profissionais de saúde na identificação de mulheres em risco de sepse e identificação precoce de sinais e sintomas, bem como predizer disfunções orgânicas e a necessidade de maior nível de cuidado. Nesse contexto, uma breve ferramenta de avaliação à beira do leito, conhecida como escore qSOFA, foi introduzida na prática clínica. O escore qSOFA avalia a presença de 3 critérios clínicos: pressão arterial sistólica menor ou igual a $100 \mathrm{mmHg}$, frequência respiratória maior ou igual a 22 por minuto e estado mental alterado (escala de coma de Glasgow). Se 2 ou mais desses critérios estiverem presentes, a paciente tem risco aumentado de sepse e pior prognóstico. Esses sinais devem levar o médico a investigar cuidadosamente a disfunção orgânica, iniciar ou intensificar a terapia, aumentar a acuidade do monitoramento e considerar a transferência para uma unidade de terapia intensiva (UTI). A pontuação qSOFA não define sepse; em vez disso, é um método rápido de identificar os pacientes 
com alto risco de desenvolver complicações graves que requerem uma terapia mais agressiva (Plante et al., 2019; Phillips \& Walsh, 2020).

Outras ferramentas de rastreamento de sepse opcionais são a SOFA, que também quantifica a temperatura, saturação de oxigênio capilar periférico (SpO), contagem de leucócitos (incluindo a porcentagem de neutrófilos imaturos) e nível de ácido láctico, e a Maternal Early Warning Trigger Tool (MEWT), que se destina a facilitar a avaliação imediata de mulheres em risco de hemorragia, hipertensão, sepse e comprometimento cardiopulmonar para garantir uma intervenção oportuna. A disfunção orgânica pode ser objetivamente definida como um aumento agudo de 2 ou mais pontos na pontuação SOFA, enquanto em indivíduos sem doença de base, o escore SOFA inicial deve ser zero. Embora os sistemas de pontuação de sepse possam ser úteis no estabelecimento de um diagnóstico rápido, esses instrumentos têm limitações e ainda podem exigir validação (Plante et al., 2019; Phillips \& Walsh, 2020).

Nesse contexto, a sepse puerperal é uma emergência clínica que requer diagnóstico e tratamento imediatos. Os objetivos do tratamento da sepse são o reconhecimento precoce e a intervenção agressiva para reduzir o risco de complicações e a mortalidade. A gestão precisa ser colaborativa e multidisciplinar para melhorar os resultados. Assim, o manejo é baseado no início rápido de terapia antimicrobiana, reposição de fluidos e suporte cardiopulmonar adequado, com correção de hipóxia e monitoramento hemodinâmico (Duan et al. 2019; García et al., 2016; Shafik et al., 2019; Phillips \& Walsh, 2020).

A Surviving Sepsis Campaign recomenda um bólus inicial de $30 \mathrm{~mL} / \mathrm{kg}$ de cristaloide, mas essa recomendação pode ser excessivamente agressiva. Apenas cerca de $50 \%$ dos pacientes sépticos hipotensos respondem a essa medida inicial. Nos que não o são, a administração agressiva pode levar ao extravasamento de líquido, à disfunção diastólica do ventrículo esquerdo a partir do edema de parede ventricular, bem como edema pulmonar, cerebral e intestinal com aumento da pressão intra-abdominal e maior mortalidade (Plante et al., 2019).

Pacientes com sepse cutânea, abscesso ou choque tóxico (erupção cutânea confluente, choque, falência de órgãos) quase sempre terão origem Gram positiva, provavelmente estafilocócica. Nesses casos, a flucloxacilina/vancomicina empírica deve ser iniciada após as culturas. Em contraste, a sepse urinária ou intestinal é predominantemente Gram negativa. Aqui, para o tratamento empírico podem ser utilizados os aminoglicosídeos/piperacilina-tazobactam ou meropenem (Morgan, 2020).

A profilaxia da trombose venosa com o uso diário de heparina de baixo peso molecular, além de dispositivos de compressão pneumática também estão indicados. Mulheres gravemente doentes correm o risco de desenvolver úlceras de estresse e podem ter risco aumentado de sangramento gastrointestinal, incluindo coagulopatia, tendo indicação de profilaxia com um inibidor da bomba de prótons ou um bloqueador de histamina (Phillips \& Walsh, 2020).

A disfunção renal está frequentemente presente em mulheres com sepse, envolvendo insuficiência da glândula adrenal, podendo necessitar de glicocorticoides em baixas doses como terapia de substituição, como a hidrocortisona. A disfunção miocárdica também é comum entre mulheres com sepse; $60 \%$ dos pacientes sépticos terão uma fração de ejeção menor que 45\%, levando à necessidade de infusão de dobutamina, junto com vasopressores para tratar a hipoperfusão contínua com baixo débito cardíaco (Plante et al., 2019; Phillips \& Walsh, 2020; Viderman et al., 2021).

\section{Conclusão}

A sepse puerperal continua a figurar como uma das principais causas de morbidade e mortalidade em todo o mundo. No que tange às principais recomendações vigentes, obteve-se um consenso em relação a definição de sepse materna, contudo vale ressaltar dificuldades ainda presentes na conceituação de sepse puerperal. Em relação aos agentes etiológicos e os fatores de risco, foram encontradas evidências bem esclarecidas. Por outro lado, a fisiopatologia é bastante incompreendida, além de se verificar grande escassez de evidências científicas atuais, sobretudo no tocante ao diagnóstico e tratamento precoces. Em suma, sabe-se que estudos mais aprofundados são necessários para melhor entendimento dessa doença, sendo necessárias 
abordagens minuciosas para sanar as diversas lacunas existentes em relação à sepse puerperal.

\section{Referências}

Baena, C. P. (2014). Meta-analysis and Systematic Review: Is it gold-standard? Rev Med UFPR, 1(2):71-74. https://doi.org/10.5380/rmu.v1i2.40706

Belarmino, A. C., Pinto, M. C. O., Frota, A. C., Alves, L. C., \& Ferreira Júnior, A. R. (2020). Perspectivas da enfermagem sobre o protocolo da sepse materna: análise à luz da teoria da complexidade. Avances en Enfermería, 38(3), 286-295. https://doi.org/10.15446/av.enferm.v38n3.84775

Céspedes-Fernández, P. L., Díaz-Martínez, A., Vázquez-Nassiff, J. J., \& Céspedes-Díaz, P. E. (2020). Factores asociados a la presentación de sepsis puerperal en provincia Guantánamo, 2017-2018. Revista Información Científica, 99(1), 12-19.

Duan, R., Xu, X., Wang, X., \& Yu, H. (2019). Perinatal outcome in women with bacterial sepsis: A cross-sectional study from West China. Medicine, 98(44):e17751. https://doi.org/10.1097/MD.0000000000017751

Foeller, M. E., Sie, L., Foeller, T. M., Girsen, A. I., Carmichael, S. L., Lyell, D. J., Lee, H. C., \& Gibbs, R. S. (2020). Risk factors for maternal readmission with sepsis. American journal of perinatology, 37(5), 453-460. https://doi.org/10.1055/s-0039-1696721

Galvão, A., Braga, A. C., Gonçalves, D. R., Guimarães, J. M., \& Braga, J. (2016). Sepsis during pregnancy or the postpartum period. Journal of obstetrics and gynaecology, 36(6), 735-743. https://doi.org/10.3109/01443615.2016.1148679

García, C. R. A., Luna, Z. M., \& Acosta, J. G. (2016). Síndrome de shock tóxico en el puerperio: Reporte de un caso. Revista de la Asociación Mexicana de Medicina Crítica y Terapia Intensiva, 30(1), 55-58.

Knight, M., Mottram, L., Gray, S., Partlett, C., Juszczak, E., \& ANODE collaborative group (2018). Prophylactic antibiotics for the prevention of infection following operative vaginal delivery (ANODE): study protocol for a randomised controlled trial. The Lancet, $19(1), 395$.

Koo, Y. J. (2018). Puerperal septic shock and necrotizing fasciitis caused by staphylococcus caprae and Escherichia coli. Yeungnam University journal of medicine, 35(2), 248-252. https://doi.org/10.12701/yujm.2018.35.2.248.

Lopez, J. P. \& Müller, E. A. (2016). Late puerperal sepsis, case report and literature review. Rev Case Rep, 2(1): 17-26.

Matos, K. L. A., Morais, L. O., Cavalcante, C. C., Nunes, E. J. G., Pereira, T. Z., Silva, L. P., Potros, F. R., Maia, P. R., \& Ribeiro, F. M. (2019). Sepse durante o período gestacional. Revista Eletrônica Acervo Saúde, 11(17): e1166. https://doi.org/10.25248/reas.e1166.2019

Mendes, S., Silveira, P., \& Galvão, M. (2008). Revisão integrativa: método de pesquisa para a incorporação de evidências na saúde e na enfermagem. Texto Contexto Enferm., 17(4): 758-64. https://doi.org/10.1590/S0104-07072008000400018

Morgan, M. (2020). Challenging infections in pregnancy. Obstetrics, gynaecology and reproductive medicine, 30(9), 289-297. https://doi.org/10.1016/j.ogrm.2020.06.005

Phillips, C., \& Walsh, E. (2020). Group A Streptococcal infection during pregnancy and the postpartum period. Nursing for women's health, 24(1), 13-23. https://doi.org/10.1016/j.nwh.2019.11.006

Plante, L. A., Pacheco, L. D., \& Louis, J. M. (2019). SMFM Consult Series \#47: Sepsis during pregnancy and the puerperium. Am J Obstet Gynecol, 220(4): B2-B10. https://doi.org/10.1016/j.ajog.2019.01.216.

Ramírez Salinas, Y., Illas, A. Z., Infante del Rey, S., Ramírez Salinas, Y. M, Castellanos, I. M., \& Mayor, V. M. (2016). Infección del sitio quirúrgico en puérperas con cesárea. Revista Cubana de Obstetricia y Ginecología, 42(1).

Shafik, S., Mallick, S., Fogel, J., Tetrokalashvili, M., \& Hsu, C. D. (2019). The utility of systemic inflammatory response syndrome (SIRS) for diagnosing sepsis in the immediate postpartum period. J Infect Public Health, 12(6), 799-802. https://doi.org/10.1016/j.jiph.2019.04.003

Silva, J. V. C. P., Santos, L. A., Pontes, L. T. A., Vasconcelos, T. H., Teodósio, D. de O., \& Melo, G. B. (2020). Fatores de risco e complicações relacionados à mortalidade materna. Caderno de Graduação, 6(2), 87-100.

Singer, M., Deutschman, C. S., Seymour, C. W., Shankar-Hari, M., Annane, D., Bauer, M., Bellomo, R., Bernard, G. R., Chiche, J.-D., Coopersmith, C. M.; Hotchkiss, R. S., Levy, M. M., Marshall, J. C., Martin, G. S., Opal, S. M., Rubenfeld, G. D., van der Poll, T., Vicent, J.-L., \& Angus, D. C. (2016). The third international consensus definitions for sepsis and septic shock (sepsis-3). JAMA, 315(8), 801-810. https://doi.org/ 10.1001/jama.2016.0287

Viderman, D., Umbetzhanov, Y., Temirov, T., \& Kuzkov, V. V. (2021). Refractory gram-negative septic shock complicated by extended purpura fulminans and multiple organ failure in a 23-year-old puerpera -a case report. Korean journal of anesthesiology, 74(3), 266-270. 